\title{
Trust and insurance
}

\section{Luigi Guiso $^{1}$}

Accepted: 1 June 2021

(c) The Geneva Association 2021

It has long been recognised that trust is a key ingredient in fostering economic and financial transactions and achieving business success. Over 50 years ago, Nobel prize winner Kenneth Arrow, after recognising the pervasiveness of mutual trust in commercial and non-commercial transactions, went so far as to state that "it can be plausibly argued that much of the economic backwardness in the world can be explained by the lack of mutual confidence". If it is indeed so pervasive, it is no wonder that trust also matters for the development of insurance markets, and in particular for financial and insurance exchanges. As Arrow noticed, while being an ingredient in most exchanges, trust is likely to be particularly important in those transactions that involve an element of time. Financial transactions, i.e. exchanges of money over time, are particularly dependent on trust. Any financial transaction, be it a loan, a purchase of a stock of a listed company, investment in the bonds of a corporation or a government, or the purchase of an insurance policy, has a fundamental characteristic: it is an exchange of money today against a promise of (more) money in the future, possibly in a particular state of the world. What leads one to believe that promise and make the exchange possible? Trust. The trust of a person who has invested in the stock of a company that the company's managers will not appropriate his money. The trust of a bank that a borrower will repay his loan as promised. The trust of a person purchasing a casualty insurance policy that the insurance company will readily pay the indemnity if the casualty occurs. But also the trust that the insurance company places in the policyholder, that they will not claim an indemnity when it is not due.

This issue collects three contributions on this key topic that shed light on various dimensions of the link between trust and insurance. While trust enters all financial transactions, it plays a specific role in insurance contracts. First, the insured, when entering the contract and paying the insurance premium upfront, has to trust that the insurance company will pay the indemnity promptly in the case the casualty actually occurs at some time in the future. Second, the insurance company has to trust that the insured, once the premium is paid, does not act so as to raise the risk of the casualty by adopting riskier behaviour. This type of risk is known as moral hazard

Luigi Guiso

luigi.guiso55@gmail.com

1 Einaudi Institute for Economics and Finance, Via Sallustiana 62, 00187 Rome, Italy 
and may be cautioned against by pricing it into the premium. Trust is also entailed because the insured can cheat the company by pretending that a casualty actually has occurred when it has not, by faking a casualty or by aggravating its consequences. The possibility of fraud implies that the willingness of an insurance company to offer insurance to a certain pool of customers also depends on its beliefs about the pool's average trustworthiness.

In sum, insurance exchanges require trust on both sides (Guiso 2012; Gennaioli et al. 2021). The trust of the buyer and seller of insurance may interact in important ways. Limited trust on the side of the insurance company vis-à-vis its customers can result in limited trust in the latter towards the company. If the company does not trust the claim of the client, it will hold on the payment of the indemnity to investigate whether it is honestly claimed; but delayed payments will disappoint clients, in turn lowering trust in the company.

Figure 1, taken from my study (Guiso 2012), documents the importance of trust for insurance demand. It shows a strong positive correlation between how much trust individuals belonging to a sample of Italian small businessmen have in insurance companies and a measure of insurance coverage of their businesses (the number of insurance policies against a set of insurable risks). The paper also documents one key source of this trust: customer satisfaction with the company, which, in turn, is affected by the behaviour of the insurance company.

As argued, insurance exchanges are best characterised as two-sided trust exchanges. Individuals can cheat the insurance company by, for instance, claiming an indemnity when it is not due. On the other hand, insurance companies can cheat individuals by not recognising an indemnity when it is honestly claimed. In

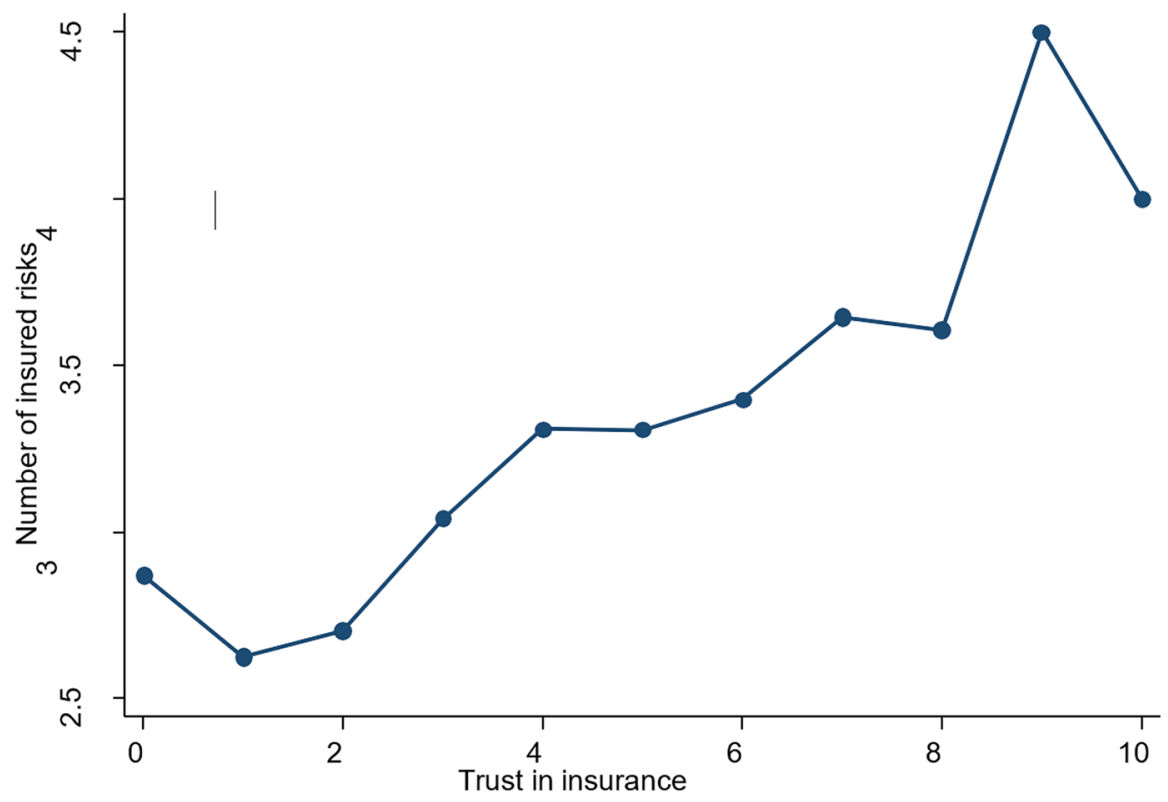

Fig. 1 Trust and the demand for insurance 
this regard, the two sides are symmetric. However, there is an important asymmetry. If it is the client that breaches trust and cheats the company, his behaviour is socially condemned and disapproved of; if it is the company that breaches trust and cheats on the clients, however, its behaviour is considered much worse by society. This is because insurance companies are much more "powerful and wealthier' than individuals and causing harm to a weaker agent is perceived as socially less acceptable than causing harm to a powerful, wealthy agent.

To sum up, trust enters insurance exchanges because the latter is a typical financial exchange that trades spot money against promises of future money. But what makes them peculiar and different from other financial exchanges is that both parties in the trade, the insurance seller and buyer, require trust to carry over the transaction. Needless to say, one could do without trust if parties could write complete insurance contracts and the latter could be perfectly enforced; however, we live in a world where contracts lack completeness and perfect enforceability in courts is a dream. These imperfections mean that trust matters.

The three contributions in this issue expand our knowledge of the role of trust in insurance markets in various directions. The first paper by Tao Sun focuses on insurance providers and asks how much insurance companies' willingness to take on risk is affected by the culture of the country in which they operate. It concentrates on two cultural dimensions: people's trust and people's risk avoidance. Using cross-country data, the article finds that companies in countries with high levels of societal trust tend to adopt riskier strategies, while the opposite is true for companies operating in countries characterised by a high degree of Hofstede risk avoidance. These results may help shed light on the observed differences in behaviour between insurance companies from culturally heterogeneous countries when entering the same market in the global economy.

The second paper by Jacob Dexe, Ulrik Franke and Alexander Rad discusses and offers evidence on a very hot topic: how reliance on new information technologies, such as the processing of large amounts of individual data and the use of artificial intelligence by the insurance industry, can affect company-client relations, in particular customers' confidence in the company. Automation and the unavoidable anonymity that it entails move the management of the relationship with the customer from man to machine. The key question is: do customers trust the machine more then they trust men? This is not an easy question and the answer may be neither general nor unequivocal. Probably, it depends, for instance, on how much information people have on the artificial intelligence algorithms and thus on transparency, on how good people are at figuring out how trustworthy humans are, or how much trust they already have in humans. The paper studies some of these issues, leveraging data from interviews with a sample of insurance professionals in Sweden who were taking a position on whether and how transparency can help deal with dilemmas that may arise due to the adoption of a new technology. It is interesting to notice that this issue emerges even in Sweden - a country with one of the highest levels of trust worldwide. Hence, the issue must be perceived as even more important in less trusting countries. On the other hand, because of this, one needs to take some care in extending the conclusions of this study to other countries. 
The paper by Marta Ostrowska, the third and last contribution to the special issue, sheds further light on the issue of the impact of new technologies on the nature of the buyer-seller relationship in insurance markets. She focuses on a specific and very important issue: risk assessment. There is no doubt that new technologies and the capacity to explore large amounts of data from various sources allow insurers to obtain much more precise client risk profiles. Sometimes, customer risk taking can be monitored at very high frequency and additional data can be collected, for instance through wearables. Needless to say, more information allows better pricing of products and can potentially expand insurance availability; however, new technologies will replace the customer as a voluntary source of information. The paper studies whether traditional policyholder risk declarations will still be needed, or whether the information gathered on the customer thanks to new technologies will be so complete so as to crowd out the need for the personal and conscious involvement of the customer in passing over to the company risk-relevant information. Technology can also potentially create a reversal in the distribution of information, making the party that was traditionally taken to be the least informed (typically the insurance company) the most informed. Companies may be able to process so many data assembled from various sources that they may know more about the customer's risk profile than the customer himself, overturning traditional information asymmetry. As one can easily guess, this opens up completely new issues and raises many questions about the validity of the traditional theoretical paradigm on which insurance theory rests.

\section{References}

Gennaioli, N., R. LaPorta, F. Lopez-de-Silanes, and A. Shleifer. 2021. Trust and insurance contracts. Review of Financial Studies.

Guiso, L. 2012. Trust and insurance markets. Economics Notes 41: 1-26.

Publisher's Note Springer Nature remains neutral with regard to jurisdictional claims in published maps and institutional affiliations.

\section{About the author}

Luigi Guiso is the Axa Professor of Household Finance at the Einaudi Institute for Economics and Finance in Rome. He has held positions at the University of Rome Tor Vergata and at the European University Institute in Florence. He has directed the Finance Programme at the Center for Economic Policy Research in London and taught as a visiting professor at the at the University of Chicago Booth School of Business, Northwestern University and Imperial College. He is a Fellow of the Econometric Society. Besides his work in the field of household finance, he has contributed research in the field of labour economics, firm investments and financial decisions, entrepreneurship and banking, political economy and institutions, and culture and economics. His research has been published in major scholarly journals such as The Review of Economic Studies, The Quarterly Journal of Economics , Journal of Political Economy, American Economic Review and Econometrica. 